\title{
Modeling and stability analysis for a varicella zoster virus model with vaccination
}

\author{
Stephen Edward, Dmitry Kuznetsov, Silas Mirau \\ School of CoCSE, Nelson Mandela African Institution of Science and Technology, P.O. Box 447, Arusha, Tanzania \\ Email address: \\ edwards@nm-aist.ac.tz (S. Edward),dmitry.kuznetsov@nm-aist.ac.tz (D. Kuznetsov), silas .mirau@nm-aist.ac.tz (S. Mirau)
}

To cite this article:

Stephen Edward, Dmitry Kuznetsov, Silas Mirau. Modeling and Stability Analysis for a Varicella Zoster Virus Model with Vaccination. Applied and Computational Mathematics. Vol. 3, No. 4, 2014, pp. 150-162. doi: 10.11648/j.acm.20140304.16

\begin{abstract}
In this paper, a deterministic mathematical model for transmission dynamics of Varicella Zoster Virus (VZV) with vaccination is formulated. The effective reproduction number is computed in order to measure the relative impact for individual or combined intervention for effective disease control. The effective reproductive number, $R_{e}$ is defined as the number of secondary cases that one infected individual will cause through the duration of the infectious period. The disease-free equilibrium is computed and proved to be locally asymptotically stable when $R_{e}<1$ and unstable when $R_{e}>1$.It is proved that there exists at least one endemic equilibrium point for all $R_{e}>1$. In the absence of diseaseinduced death, it is proved that the transcritical bifurcation at $\mathrm{R}_{0}=1$ is supercritical (forward). Sensitivity analysis is performed on the basic reproduction number and it is noted that the most sensitive parameters are the probability of transmission of the disease from an infectious individual to a susceptible individual per contact, $\beta$, per capita contact rate , $c$, per capita birth rate, $\pi$ and the progression rate from latent to infectious stage, $\delta$. Numerical simulations of the model show that, the combination of vaccination and treatment is the most effective way to combat the epidemiology of VZV in the community.
\end{abstract}

Keywords: Modeling, Sensitivity, Treatment, Vaccination, Epidemiology

\section{Introduction}

Chickenpox (also called varicella) is a disease caused by virus known as varicella -zoster virus (VZV) also known as human herpes virus 3 (HHV -3)[1]. In non-vaccinated populations, primary infections tend to occur at a younger age[2].

The main symptoms of chickenpox are fever, headache, stomach ache,itchy rash especially in the mouth, on chest, abdomen, back, face and upper arms and legs. The rash starts as small red spot, difficult breathing, malaise and anorexia.

Chickenpox is widely transmitted from touching the fluids from chickenpox blister. The virus is spread either by direct contact with a person with active chickenpox or shingles, or by direct contact with clothes or other articles infected with vesicle fluid, saliva, nasal discharge, or by air borne spread of small droplets of infected mucous of fluid.

There are different notions to the origin of the name of this disease. One is, once infected; the skin appeared as picked by chicken. Another is the rash resembles the seeds of Chick Peas. Most common explanation is that the disease is not that much dangerous compared to small pox so it is a 'chicken' version of pox.

The occurrence of chickenpox is different in different geographical zones. In temperate countries chickenpox is usually a mild, self-limiting infection, affecting pre-school children [3], however, the incidence of chickenpox in these areas is increasing in adolescents and adults [4], which may in part be due to increased world travel and economic migration of susceptible individuals. In many tropical countries the epidemiology is different, with about $60 \%$ of adults being immune [5].

In the past, the varicella zoster virus affected virtually the entire population and had substantial morbidity and mortality associated with both primary varicella and herpes zoster reactivation. Since the varicella vaccine was first approved in 1995, there has been a significant decline in incidence, morbidity, and mortality caused by primary varicella. Breakthrough disease with the one-dose vaccine schedule led to the recommendation in 2006 that children receive a two-dose vaccine series. Older adults have also benefited from the development of the zoster vaccine. In 2006, the Food and Drug Administration approved the 
zoster vaccine, a higher concentration of the same live attenuated virus used in the primary varicella vaccine, for persons 60 years of age or older. It has the potential to help millions of people to avoid the pain associated with reactivation of the varicella zoster virus by reducing the incidence and severity of herpes zoster and postherpetic neuralgia.

The treatment for a patient with chickenpox is: reducing itches and irritation by keeping the skin cool with light clothing and tepid baths or sponging. Calamine lotion applied to spots, or antihistamine tablets may also help. Paracetamol or ibuprofen can be taken if lesions are painful and will lose fever.

The first vaccination was developed by Michiaki Takahash in 1974 derived from Oka strain. Some countries require the varicella vaccine for children before entering elementary school. Immunity derived from vaccine is not lifelong and subsequent vaccination is necessary usually after 5 years after initial vaccination. Chickenpox is characterized by long latent period (about two weeks), infectious period (one week) and permanent immunity after recovery.

[6] were among the first to explore the relationship between varicella and zoster using mathematical models; they examined the impact of vaccination on long-term equilibrium incidence of these diseases, but ignored the short medium term.

[7] examined the possible influence of zoster on the transmission dynamics of varicella, but did not investigate the impact of vaccination on the incidence of zoster (their model assumes a constant background force of infection of zoster which remains unchanged through time).Two possible dangers of an extensive varicella vaccination program are more varicella (chickenpox) cases in adults, when the complication rates are higher, and an increase in cases of zoster (shingles) [8].

Since almost all children become infected with VZV, the annual incidence of varicella is approximately equal to the birth rate. At least $90 \%$ of adults in the United States are immune to $\mathrm{VZV}$, but rates of immunity to $\mathrm{VZV}$ in adults can be as low as $50 \%$ in tropical areas [9]. Following a varicella infection, VZV is established in a latent form in the dorsal root ganglia by an ascending infection along sensory nerves from the skin. This latent herpes virus becomes reactivated during the lifetimes of about $15 \%$ of those who had a primary varicella infection, causing zoster (commonly known as shingles), a painful vesicular rash appearing along one or two of the sensory root nerves. Reactivation correlates with diminished VZV cell-mediated immunity, so that zoster develops more frequently among people immunocompromised by age, disease, or therapy. Thus zoster occurs at all ages, but is more common among the elderly. Transmission of VZV from people with zoster can occur, but is much less likely than from people with primary varicella $[9,10,11]$

Primary infection by varicella-zoster virus (VZV) causes the clinical syndrome 'chickenpox' (CP), mainly in childhood. An effective commercial childhood CP vaccine has been available for nearly 20 years. It is recommended to be used in a two-dose schedule because experience with a single dose has led to frequent (milder) breakthrough infections [12]. After primary infection VZV remains latent in neural ganglia until reactivation. Herpes zoster (HZ), also called shingles, is caused by the symptomatic reactivation of VZV and this reactivation is assumed to be a consequence of a lower cellular immunity mainly in immunocompromised or older individuals [13, 14, 15].

[16] Developed a mathematical model on perspective on the impact of varricella immunization on Herpes zoster. Their model was a multi-country model of VZV transmission and reactivation, which they used to evaluate the possible impact of varicella vaccination on HZ (Herpes Zoster) epidemiology in Italy, Finland and UK.

Despite large uncertainties their finding might provide explanations for ambiguous empirical evidences about increases of $\mathrm{HZ}$ in those cities where mass vaccination is ongoing.

The proposed Multi-country perspective model shows, under different vaccination scenarios, that an increase in $\mathrm{HZ}$ incidence is not a certain fact, but rather seems to depend on the presence or absence of factors promoting a strong boosting intensity and that may or may not ,be heavily affected by changes in varicella circulation due to introduction of immunization programs.

In this study we formulate a deterministic mathematical model for transmission dynamics of Varicella Zoster Virus (VZV).The model to be developed considers a total population to be non-constant and non-age structured. It should be clearly known that we are interested to investigate only primary Varicella even though we know from literature $[9,10,11]$ that reactivation of VZV can lead to a disease called zoster which is less severe as compared to varicella (chickenpox) and mostly occurs in adulthood. The model will further take account of two dose vaccine coverage. Stability analysis, simulation, sensitivity analysis will be carried out in this paper.

\section{Model Formulation}

In this section we formulate a deterministic mathematical model for VZV which incorporates vaccination strategy. The total population is divided into the following epidemiological classes or subgroups: susceptible $S$, vaccinated $V$, Exposed $E$, infectious $I$, recovered $R$. Basically; we modify the SEIR model by adding a vaccination compartment which caters for immunization.

Let us assume that the per capita birth rate $\pi$ is constant, the natural fatality rate $\mu$ is time constant, there is no disease induced death, the members of the population mix homogenously (have the same interactions with one another to the same degree), and assume that on recovery, there is a permanent immunity of the rate $\eta$. Furthermore, assume that individuals can be infected through direct $\operatorname{contact} c$, with an infectious individual. We let $\beta$ to be the 
probability that a susceptible individual becomes infected by one infectious individual. We also let $\Lambda$ be the constant recruitment rate.

The susceptible and vaccinated individuals are recruited by both birth and immigration. A proportion $\rho$ of the recruits are vaccinated, the remaining $1-\rho$ are not vaccinated so they join the susceptible compartment. Proportions of newborns $\phi$ are vaccinated, and the remaining $1-\phi$ newborns are not vaccinated and hence join the susceptible compartment. We consider that a proportion of the population of susceptible to receive a first dose vaccine at the rate $\theta_{1}$, whereas the rest of it progress with the disease.

The primary vaccine wanes at the rate $\alpha$ after a fixed time t. After the first vaccine has expired, a proportion $1-f$ of the vaccinated individuals at dose one, join the susceptible compartment at the rate $\alpha$ while the remaining proportion $f$ receive a second dose at the rate $\theta_{2}$. Our assumption is that the individuals who have attended the first and the second dose consecutively receive permanent immunity; otherwise they become susceptible to the disease again. The susceptible individuals enter the exposed compartment at the rate $\lambda$ which is a force of infection. The exposed individuals are the ones who are infected but not infectious. After some time the exposed become infectious, they move from exposed state to infectious at the rate $\delta$. An infected individual recover at rate $\eta$, and according to the nature of the disease; the recovered individuals are permanently immune.

This description of dynamics of VZV can be summarized by compartmental diagram Figure 1

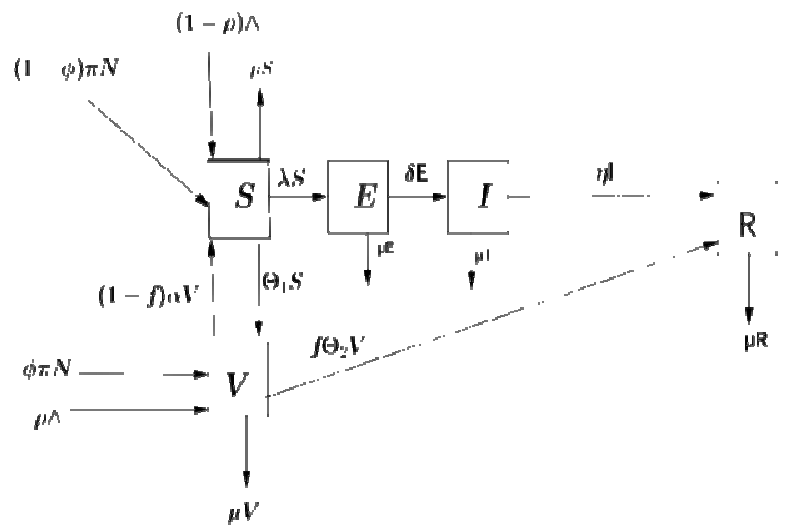

Figure 1. A Compartmental diagram for the dynamics of Varicella Zoster Virus (VZV) in a community with Immunization.

Table 1. Parameters and their description.

\begin{tabular}{ll}
\hline Parameter & Description \\
\hline$\alpha$ & The rate of waning of a vaccine \\
$\beta$ & $\begin{array}{l}\text { Probability of one infected individual to become } \\
\delta\end{array}$ \\
$\phi$ & Progressious rate from latent to infectious \\
$\rho$ & Proportions of newborns who are vaccinated. \\
\hline
\end{tabular}

\begin{tabular}{ll}
\hline Parameter & Description \\
\hline$a$ & Arrival rate. \\
$c$ & Per capita contact rate \\
$\theta_{1}$ & Fraction of individuals who receive a first dose \\
$\theta_{2}$ & vaccine \\
$\mu$ & Rate at which of individuals receive a second \\
$\pi$ & dose vaccine \\
$\eta$ & Per capita natural mortality rate \\
$\Lambda$ & Per capita birth rate \\
$f$ & Recovery rate of treated infectious individuals \\
& The recruitment rate of susceptible population \\
\hline
\end{tabular}

\subsection{The Model Equations}

From the assumptions and the dynamics between the compartments shown in the model compartments in Figure1, the impact of immunization on the epidemiology of VZV is modeled by following system of ordinary differential equations

$$
\begin{gathered}
\frac{d S}{d t}=(1-\phi) \pi N+(1-\rho) \Lambda+(1-f) \alpha V-(\lambda+\mu+\theta) S \\
\frac{d V}{d t}=\rho \Lambda+\phi \pi N+\theta_{1} S-\left((1-f) \alpha+f \theta_{2}+\mu\right) V \\
\frac{d E}{d t}=\lambda s-(\mu+\delta) E \\
\frac{d I}{d t}=\delta E-(\eta+\mu) I \\
\frac{d R}{d t}=\eta I+f \theta_{2} \mathrm{v}-\mu R
\end{gathered}
$$

where $\lambda$ is the force of infection and is given by $\lambda=\frac{\beta c I}{N}$. The total population size is

$$
N(t)=S(t)+V(t)+E(t)+I(t)+R(t)
$$

where, adding Equation of the system (1-5). We get

$$
\frac{d N}{d t}=\Lambda+(\pi-\mu) N
$$

\subsection{Dimensionless Transformation}

For simplicity of analysis we normalized quantities. This can be done by scaling the population of each class by the total population.

$$
\text { We transform follows } v=\frac{V}{N}, s=\frac{S}{N}, e=\frac{E}{N}, i=\frac{I}{N}
$$

and $r=\frac{R}{N}$ in classes $V, S, E, I$ and $R$.

Hence the normalized model system becomes, 


$$
\begin{gathered}
\frac{d s}{d t}=(1-\phi) \pi+(1-\rho) a+(1-f) \alpha v-\left(\lambda+\theta_{1}+a+\pi\right) s \\
\frac{d v}{d t}=\phi \pi+\rho a+\theta_{1} s-\left((1-f) \alpha+f \theta_{2}+a+\pi\right) \\
\frac{d e}{d t}=\lambda s-(\delta+a+\pi) e \\
\frac{d i}{d t}=\delta e-(\eta+a+\pi) i \\
\frac{d r}{d t}=\eta i+f \theta_{2} v-(a+\pi) r
\end{gathered}
$$

Subject to the restriction that leads to studying the system (6-10) in the region $T$ where

$$
T=\left\{(s, v, e, i, r) \in R_{+}^{5}: 0 \leq s, 0 \leq v, 0 \leq e, 0 \leq i, 0 \leq r, s+v+e+i+r \leq 1\right\}
$$

The feasible region (where the model makes biological sense) can be shown to be positively invariant.

\section{Model Analysis}

The model system (6-10) is analyzed qualitatively to get insights into its dynamical features which give better understanding of the impact of immunization on the epidemiology of varicella zoster virus.

\subsection{Disease Free Equilibrium (DFE), $P_{0}$}

The disease free equilibrium of the model system (6-10) is obtained by setting $\frac{d v}{d t}=\frac{d s}{d t}=\frac{d e}{d t}=\frac{d i}{d t}=\frac{d r}{d t}=0$ and $P_{0}$ of the model system (6-10) exists and is given by:

$$
P_{0}\left(s^{*}, v^{*}, e^{*}, i^{*}, r^{*}\right)=\left(s_{0}^{*}, v_{0}^{*}, 0,0, r_{0}^{*}\right)
$$

in case there is no disease; $e=i=0$ the sum of susceptible and vaccinated populations is equal to total population. That is to say $s_{0} *+v_{0} *+r_{0}^{*}=1$

Consequently, system (6-10) is reduced to:

$$
\begin{gathered}
-\left(\lambda+\theta_{1}+a+\pi\right) s+(1-f) \alpha v+(1-\phi) \pi+(1-\rho) a=0 \\
\theta_{1} s-\left((1-f) \alpha+f \theta_{2}+a+\pi\right) v+\phi \pi+\rho a=0 \\
f \theta_{2} v-(a+\pi) r=0
\end{gathered}
$$

which implies:

$$
\begin{gathered}
s_{0}^{*}=\frac{(1-f) \alpha(a+\pi)+\left(f \theta_{2}+a+\pi\right)[(1-\phi) \pi+(1-\rho) a]}{(a+\pi)(1-f) \alpha+\left(f \theta_{2}+a+\pi\right)\left(\theta_{1}+a+\pi\right)} \\
v_{0}^{*}=\frac{(a+\pi)\left(\theta_{1}+\phi \pi+\rho a\right)}{\theta_{1}\left(f \theta_{2}+a+\pi\right)+(a+\pi)\left[(1-f) \alpha+f \theta_{2}+a+\pi\right]}
\end{gathered}
$$

$$
r_{0}^{*}=\frac{f \theta_{2}\left(\theta_{1}+\phi \pi+\rho a\right)}{\theta_{1}\left(f \theta_{2}+a+\pi\right)+(a+\pi)\left[(1-f) \alpha+f \theta_{2}+a+\pi\right]}
$$

Thus the Disease Free Equilibrium (DFE) point denoted by $P_{0}$ of the model system (6-10) exists and is given by:

$$
P_{0}\left(s^{*}, v^{*}, e^{*}, i^{*}, r^{*}\right)=\left(s_{0}^{*}, v_{0}^{*}, 0,0, r_{0}^{*}\right)
$$

\subsection{The Basic Reproduction Number, $R_{0}$}

[17] defined the basic reproduction number denoted by $\mathrm{R}_{0}$, as the average number of secondary infections caused by an infectious individual during his or her entire period of infectiousness. The basic reproduction number is an important non-dimensional quantity in epidemiology as it sets the threshold in the study of a disease both for predicting its outbreak and for evaluating its control strategies. Thus, whether a disease becomes persistent or dies out in a community depends on the value of the reproduction number, $R_{0}$.

Furthermore, stability of equilibria can be analyzed using $R_{0}$. If $R_{0}<0$ it means that every infectious individual will cause less than one secondary infection and hence the disease will die out and when $R_{0}>1$, every infectious individual will cause more than one secondary infection and hence the disease will invade the population. A large number of $R_{0}$ may indicate the possibility of a major epidemic. For the case of a model with a single infected class, $R_{0}$ is simply the product of the infection rate and the mean duration of the infection.

In more complicated epidemics we compute the basic reproduction number, $R_{0}$ using the next generation operator approach by [18]

From the system Equations (6-10) we define $\mathcal{F}_{i}$ and $\mathcal{V}_{i}$ as

$$
\mathcal{F}_{i}=\left[\begin{array}{c}
\beta c i s \\
0
\end{array}\right], \mathcal{V}_{i}=\left[\begin{array}{c}
(\delta+a+\pi) e \\
\delta e-(\eta+a+\pi) i
\end{array}\right]
$$

We differentiate $\mathcal{F}_{i}$ with respect to $e$ and $i$ to get

$$
F=\left[\begin{array}{cc}
0 & \beta c s \\
0 & 0
\end{array}\right]
$$

We differentiate $\mathcal{V}_{i}$ with respect to $e$ and $i$ and get $V$

$$
V=\left[\begin{array}{cc}
(\delta+a+\pi) & 0 \\
-\delta & (\eta+a+\pi)
\end{array}\right]
$$

We find the inverse of $V$ and get

$$
V^{-1}=\left[\begin{array}{cc}
\frac{1}{\delta+a+\pi} & 0 \\
\frac{\delta}{(\delta+a+\pi)(\eta+a+\pi)} & \frac{1}{\eta+a+\pi}
\end{array}\right]
$$




$$
\begin{gathered}
F V^{-1}=\left[\frac{\partial \mathcal{F}_{i}\left(E_{0}\right)}{\partial x_{j}}\right]\left[\frac{\partial \mathcal{V}_{i}\left(E_{0}\right)}{\partial x_{j}}\right]^{-1} \\
V^{-1}=\left[\begin{array}{ccc}
0 & \beta c s_{0} * \\
0 & 0
\end{array}\right]\left[\begin{array}{cc}
\frac{1}{\delta+a+\pi} & 0 \\
\frac{\delta}{(\delta+a+\pi)(\eta+a+\pi)} & \frac{1}{\eta+a+\pi}
\end{array}\right] \\
=\left[\begin{array}{cc}
\frac{\beta c s_{0} * \delta}{(\delta+a+\pi)(\eta+a+\pi)} & \frac{\beta c s_{0} *}{\eta+a+\pi} \\
0 & 0
\end{array}\right]
\end{gathered}
$$

The eigenvalues, $\lambda$ of equation (12) can be computed from the characteristic equation:

$\left|F V^{-1}-\lambda I\right|=0$.And we see that from our matrix that

$$
R_{e}=\frac{\beta c \delta\left\{(1-f) \alpha(\phi \pi+\rho a)+\left((1-f) \alpha+f \theta_{2}+a+\pi\right)((1-\phi) \pi+(1-\rho) a)\right\}}{\left\{\theta_{1}\left(f \theta_{2}+a+\pi\right)+(a+\pi)\left[(1-f) \alpha+f \theta_{2}+a+\pi\right]\right\}(\delta+a+\pi)(\eta+a+\pi)}
$$

When there is no any control strategy, then $\theta_{1}=\theta_{2}=\phi=\rho=0$

and hence $f=0, \alpha=0$, so we get basic reproduction number

$$
R_{0}=\frac{\beta c \delta}{(\delta+a+\pi)(\eta+a+\pi)}
$$

\subsection{Local Stability of the Disease-Free Equilibrium}

$$
J_{E}=\left[\begin{array}{ccccc}
-\left(\beta c i+\theta_{1}+a+\pi\right) & (1-f) \alpha & 0 & -\beta c s & 0 \\
\theta_{1} & -\left((1-f) \alpha+f \theta_{2}+a+\pi\right) & 0 & 0 & 0 \\
\beta c i & 0 & -(\delta+a+\pi) & \beta c s & 0 \\
0 & 0 & \delta & -(\eta+a+\pi) & 0 \\
0 & f \theta_{2} & 0 & \eta & -(a+\pi)
\end{array}\right]
$$

Here we investigate the local stability of the disease-free equilibrium point,

$$
P_{0}\left(s^{*}, v^{*}, e^{*}, i^{*}, r^{*}\right)=\left(s_{0}^{*}, v_{0}^{*}, 0,0, r_{0}^{*}\right) .
$$

Thus, we linearize model system (6-10) by computing its Jacobian matrix, $J_{E}$. The Jacobian matrix is computed by differentiating each equation in the system with respect to the state variables $s, v, e, i, h, r$.

at DFE

$$
\operatorname{Det}\left(J_{E_{0}}\right)=\left|\begin{array}{ccccc}
-\left(\theta_{1}+a+\pi\right) & (1-f) \alpha & 0 & -\beta c s_{0} & 0 \\
\theta_{1} & -\left((1-f) \alpha+f \theta_{2}+a+\pi\right) & 0 & 0 & 0 \\
0 & 0 & -(\delta+a+\pi) & \beta c s_{0} & 0 \\
0 & 0 & \delta & -(\eta+a+\pi) & 0 \\
0 & f \theta_{2} & 0 & \eta & -(a+\pi)
\end{array}\right|
$$

Then, the local stability of $P_{0}$ is determined by the trace method, based on the Jacobian matrix (16). The diseasefree equilibrium point, $(P 0)$ is locally asymptotically stable if trace $\operatorname{Tr}\left(J_{E_{0}}\right)<0$ and $\operatorname{Det}\left(J_{E_{0}}\right)>0$ otherwise it is unstable.

So we get

$$
\operatorname{Tr}\left(J_{E_{0}}\right)=-\left(\theta_{1}+5 a+5 \pi+(1-f) \alpha+f \theta_{2}+\eta\right)<0
$$

$\operatorname{Det}\left(J_{E_{0}}\right)=(a+\pi)(\eta+a+\pi)(\delta+a+\pi)\left\{\left(\theta_{1}+a+\pi\right)((1-f) \alpha+\right.$

$$
\left.\left.f \theta_{2}+a+\pi\right)+\alpha \theta_{1}(1-f)\right\}\left[1-\frac{\beta c \delta s_{0}}{(\eta+a+\pi)(\delta+a+\pi)}\right]
$$

But

$$
R_{e}=\frac{\beta c \delta s_{0}}{(\eta+a+\pi)(\delta+a+\pi)}
$$

So we get

$$
(a+\pi)(\eta+a+\pi)(\delta+a+\pi)\left(1-R_{e}\right)\left\{\left(\theta_{1}+a+\pi\right)\left((1-f) \alpha+f \theta_{2}+a+\pi\right)+\alpha \theta_{1}(1-f)\right\}>0
$$


provided $R_{e}<1$

Since $\operatorname{Tr}\left(J_{E}\right)<0$ and $\operatorname{Det}\left(J_{E}\right)>0$

Then a DFE is asymptotically stable otherwise it is unstable if $R_{e}>1$.

Thus we have established the following lemma.

Lemma

The disease-free equilibrium $\mathrm{P}_{0}$ is locally stable if $R_{e}<1$ and unstable if $R_{e}>1$. The quantity $R_{e}$ is the effective basic reproduction number of the disease. It represents the average number of new infections produced by one infected individual introduced in a susceptible population. It is a useful quantity in the study of a disease as it sets the threshold for its establishment. If $R_{e}<1$ then, the disease-free equilibrium is locally stable.

\subsection{Global Stability of Disease Free Equilibrium Point (DFE)}

In this section, we analyze the global stability of disease -free steady state. Here we use the method developed by [19]. Now we state two conditions which guarantee the global stability of the disease free state. We rewrite the model system (6-10) as

$$
\begin{aligned}
& \frac{d X}{d t}=F(X, Z), \\
& \frac{d Z}{d t}=G(X, Z), G(X, 0)=0,
\end{aligned}
$$

where $X=(S)$ and $Z=(E, I)$, with $X \in \mathbb{R}$ denotes the number of uninfected individuals and $Z \in \mathbb{R}^{2}$ denoting (its components) the number of infected individuals including latent and infectious. The disease-free equilibrium is now denoted by $Q_{0}=\left(X^{0}, 0\right)$ The following conditions $\left(H_{1}\right)$ and $\left(\mathrm{H}_{2}\right)$ must be met to guarantee a local asymptotic stability:

$\left(H_{1}\right)$ for $\frac{d X}{d t}=F\left(X^{0}, 0\right), X^{0}$ is globally asymptotically stable (g.a.s), $G(X, Z)=A Z-G(X, Z),\left(H_{2}\right)$ where $G(X, Z) \geq 0$ for

$$
(X, Z) \in \Omega
$$

Where $A=D_{z} G\left(X^{0}, 0\right)$ is an $M$-matrix (the off-diagonal elements of $A$ are non-negative) and $\Omega$ is the region where the model makes biological sense.Then the following lemma holds:

Lemma 1

The fixed point $Q_{0}=\left(X^{\theta}, 0\right)$ is globally asymptotic stable (g.a.s) equilibrium of system (6-10) provided that $R_{e}<1$ (l.a.s) and that the assumptions $\left(H_{1}\right)$ and $\left(H_{2}\right)$ are satisfied.

We state the following theorem:

Theorem 1

Suppose $R_{e}<1$. The disease free equilibrium $P_{0}$ is globally asymptotically stable.

Proof.
The system equation (6-10) can be expressed in the form of equation (17) and thus we get

$$
\begin{gathered}
X=(s, r), Z=(e, i), \\
A=\left(\begin{array}{cc}
-(\delta+a+\pi) & \beta_{s}^{*} \\
\delta & -(\eta+a+\pi)
\end{array}\right) \\
G=\left(\begin{array}{cc}
-(\delta+a+\pi) & \beta_{S} \\
\delta & -(\eta+a+\pi)
\end{array}\right)
\end{gathered}
$$

We need to show that $\left(\mathrm{H}_{2}\right)$ holds in the system equation(6-10).

$$
\begin{aligned}
& G(X, Z)=A Z-G(X, Z), \\
& \Rightarrow G(X, Z)=A Z-G(X, Z), \\
& \hat{G}=\left(\begin{array}{cc}
-(\delta+a+\pi) & \beta s^{*} \\
\delta & -(\eta+a+\pi)
\end{array}\right)\left(\begin{array}{l}
e \\
i
\end{array}\right)- \\
& \left(\begin{array}{cc}
-(\delta+a+\pi) & \beta s \\
\delta & -(\eta+a+\pi)
\end{array}\right)\left(\begin{array}{l}
e \\
i
\end{array}\right) \\
& =\left(\begin{array}{cc}
0 & \beta\left(s^{*}-s\right) \\
0 & 0
\end{array}\right)\left(\begin{array}{l}
e \\
i
\end{array}\right) \\
& =\left(\begin{array}{c}
\beta\left(s^{*}-s\right) i \\
0
\end{array}\right)
\end{aligned}
$$

since $s^{*} \geq s$ then $\widehat{G} \geq 0$ therefore $\left(H_{1}\right)$ and $\left(H_{2}\right)$ are satisfied. Hence the Disease free equilibrium point is globally asymptotically stable provided $R_{e}<1$.

\subsection{Existence and Local Stability of Endemic Equilibrium (EE) Point}

In the presence of infection, that is $e^{*} \neq 0, i^{*} \neq 0$ the model system (6-10) has a non-trivial equilibrium point, $P$ called the endemic equilibrium point which is given by

$$
\begin{gathered}
P=\left(s^{*}, v^{*}, e^{*}, i^{*}, h^{*}, r^{*}\right) \neq 0, \\
\left(s^{*}, v^{*}, e^{*}, i^{*}, h^{*}, r^{*}\right)>0
\end{gathered}
$$

We solve for $P=\left(s^{*}, v^{*}, e^{*}, i^{*}, h^{*}, r^{*}\right) \neq 0$ from system equation (6-10) we get

$$
\begin{gathered}
v^{*}=\frac{(\phi \pi+\rho a) \lambda+(a+\pi)\left(\theta_{1}+\phi \pi+\rho a\right)}{\left((1-f) \alpha+f \theta_{2}+a+\pi\right) \lambda+\left[(a+\pi)(1-f) \alpha+\left(f \theta_{2}+a+\pi\right)\left(\theta_{1}+a+\pi\right)\right]} \\
e^{*}=\frac{\lambda s^{*}}{(\delta+a+\pi)} \\
i^{*}=\frac{\delta \lambda s^{*}}{(\delta+a+\pi)(\eta+a+\pi)} \\
r^{*}=\frac{\eta i^{*}+f \theta_{2} v^{*}}{(a+\pi)}
\end{gathered}
$$

Since each of these variable is a function of force of 
infection, we know $i^{*}$ in terms of force of infection is

$$
i^{*}=\frac{\delta \lambda s *}{(\delta+a+\pi)(\eta+a+\pi)}
$$

where

$$
s^{*}=\frac{(1-f) \alpha(a+\pi)+\left(f \theta_{2}+a+\pi\right)[(1-\phi) \pi+(1-\rho) \alpha]}{\left((1-f) \alpha+f \theta_{2}+a+\pi\right) \lambda+\left[(a+\pi)(1-f) \alpha+\left(f \theta_{2}+a+\pi\right)(\theta+a+\pi)\right]}
$$

It can be noticed that we can express $\mathrm{s}^{*}$ as

$$
s^{*}=\frac{k_{1}}{k_{2} \lambda+k_{3}}
$$

Where

$$
\begin{gathered}
k_{1}=(1-f) \alpha(a+\pi)+\left(f \theta_{2}+a+\pi\right)[(1-\phi) \pi+(1-\rho) a] \\
k_{2}=\left((1-f) \alpha+f \theta_{2}+a+\pi\right) \\
k_{3}=(a+\pi)(1-f) \alpha+\left(f \theta_{2}+a+\pi\right)\left(\theta_{1}+a+\pi\right) \\
i^{*}=\frac{\delta}{(\eta+a+\pi)} \cdot \frac{\lambda s^{*}}{(\delta+a+\pi)}
\end{gathered}
$$

Therefore becomes

$$
\Rightarrow i^{*}=\frac{\delta}{(\eta+a+\pi)} \cdot \frac{\lambda}{(\delta+a+\pi)} \cdot \frac{k_{1}}{\left(k_{2} \lambda+k_{3}\right)}
$$

If we substitute the expressions for $i^{*}$ from above, in the equation $\lambda=\beta c i *$, the endemic should

$$
\begin{gathered}
\beta c i^{*}=\frac{\beta c \delta}{(\eta+a+\pi)} \cdot \frac{\lambda}{(\delta+a+\pi)} \cdot \frac{k_{1}}{\left(k_{2} \lambda+k_{3}\right)}=\lambda \\
\frac{\beta c \delta}{(\eta+a+\pi)} \cdot \frac{\lambda}{(\delta+a+\pi)} \cdot \frac{k_{1}}{\left(k_{2} \lambda+k_{3}\right)}=\lambda
\end{gathered}
$$

Also

$$
k_{4}=\frac{\beta c \delta}{(\eta+a+\pi)(\delta+a+\pi)}
$$

Let

So we get

$$
k_{2} \lambda^{2}+\left(k_{3}-k_{1} k_{4}\right) \lambda=0
$$

which is a particular case of

$$
\lambda * f\left(\lambda^{*}\right)=\lambda *\left(A \lambda *^{2}+B \lambda *+C\right)=0
$$

with

$A=k_{2}>0$ since $0<f<1$ and all the remaining parameters are assumed to be positive.

$$
B=k_{3}-k_{1} k_{4}=k_{3}\left(1-\frac{k_{1} k_{4}}{k_{3}}\right)
$$

$$
C=0
$$

From

$$
s=\frac{k_{1}}{k_{2} \lambda+k_{3}}
$$

At DFE

$$
\begin{gathered}
\lambda=0, s_{0}=\frac{k_{1}}{k_{3}} \\
B=k_{3}-k_{1} k_{4}=k_{3}\left(1-\frac{k_{1} k_{4}}{k_{3}}\right)=k_{3}\left(1-\frac{\beta c \delta s_{0}}{(\eta+a+\pi)(\delta+a+\pi)}\right)
\end{gathered}
$$

recall that

$$
R_{e}=\frac{\beta c \delta s_{0}}{(\eta+a+\pi)(\delta+a+\pi)}
$$

therefore

$$
B=k_{3}\left(1-R_{e}\right)>0 \text { only if } R_{e}<1
$$

and $C=0$

The solutions of equation $\lambda^{*}=0$ of equation (19) corresponds to the disease-free equilibrium point and $f\left(\lambda^{*}\right)=0$ corresponds to a situation when the disease persists (endemic).

If we examine the quadratic critically we note that $\mathrm{A}$ is always positive and $\mathrm{B}$ is positive if $R_{e}<1$ and negative if $R_{e}>1$.From this result we state the following theorem which will be proved by using bifurcation diagram and Centre Manifold theorem.

Theorem2

The unique endemic equilibrium $E^{*}$ exists

If $A>0, B<0$ and $R_{0}>1$ and is locally asymptotic stable otherwise it is unstable if $R_{0}<1$

\subsection{Stability Analysis Using Bifurcation Analysis}

Endemic equilibrium points are steady state solutions where the disease persists in the population (all states variables are positive). We use general bifurcation theory to prove the existence of at least one endemic equilibrium point for all $R_{0}>1$ [20]. When, $R_{0}>1$ it is expected that the disease would be able to invade in the case of a backward bifurcation [21]

The center manifold theory by [22] can be used to analyze the stability near the DFE and $R_{0}>1$.

Recall

$R_{e}=\frac{\beta c \delta\left\{(1-f) \alpha(\phi \pi+\rho a)+\left[(1-f) \alpha+f \theta_{2}+a+\pi\right][(1-\phi) \pi+(1-\rho) a]\right\}}{\left\{\theta_{1}\left(f \theta_{2}+a+\pi\right)+(a+\pi)\left[(1-f) \alpha+f \theta_{2}+a+\pi\right]\right\}(\delta+a+\pi)(\eta+a+\pi)}$

Let $\psi=\beta$ be a bifurcation parameter and $R_{e}=1$ be a bifurcation point ,then 


$$
1=\frac{\psi \delta\left\{(1-f) \alpha(\phi \pi+\rho a)+\left[(1-f) \alpha+f \theta_{2}+a+\pi\right][(1-\phi) \pi+(1-\rho) a]\right\}}{\left\{\theta_{1}\left(f \theta_{2}+a+\pi\right)+(a+\pi)\left[(1-f) \alpha+f \theta_{2}+a+\pi\right]\right\}(\delta+a+\pi)(\eta+a+\pi)}
$$

and from it

$$
\psi=\frac{\left\{\theta_{1}\left(f \theta_{2}+a+\pi\right)+(a+\pi)\left[(1-f) \alpha+f \theta_{2}+a+\pi\right]\right\}(\delta+a+\pi)(\eta+a+\pi)}{\delta\left\{(1-f) \alpha(\phi \pi+\rho a)+\left[(1-f) \alpha+f \theta_{2}+a+\pi\right][(1-\phi) \pi+(1-\rho) a]\right\}}
$$

Let $s=x_{1}, v=x_{2}, e=x_{3}, i=x_{4}, r=x_{5}$

Thus the model equation (6-10) becomes

$$
\begin{aligned}
& \frac{d x_{1}}{d t}=f_{1}=(1-\Phi) \pi+(1-\rho) a+(1-f) \alpha x_{2}-\left(\psi x_{4}+\theta_{1}+a+\pi\right) x_{1} \\
& \frac{d x_{2}}{d t}=f_{2}=\Phi \pi+\rho a+\theta_{1} x_{1}-\left((1-f) \alpha+f \theta_{2}+a+\pi\right) x_{2} \\
& \frac{d x_{3}}{d t}=f_{3}=\psi x_{1} x_{4}-(\delta+a+\pi) x_{3} \\
& \frac{d x_{4}}{d t}=f_{4}=\delta x_{3}-(\eta+a+\pi) x_{4} \\
& \frac{d x_{5}}{d t}=f_{5}=\eta x_{4}+f \theta_{2} x_{2}-(a+\pi) x_{5}
\end{aligned}
$$

And the jacobian matrix of system (20) at the equilibrium is given by

$$
J_{\left(E_{0}\right)}=\left[\begin{array}{ccccc}
-(\theta+a+\pi) & (1-f) \alpha & 0 & -\psi \kappa_{1} & 0 \\
\theta & -\left((1-f) \alpha+f \theta_{2}+a+\pi\right) & 0 & 0 & 0 \\
0 & 0 & -(\delta+a+\pi) & \psi x_{1} & 0 \\
0 & 0 & \delta & -(\eta+a+\pi) & 0 \\
0 & f \theta_{2} & 0 & \eta & -(a+\pi)
\end{array}\right]
$$

From equation (21), it can be shown that the Jacobian matrix has right eigenvalues given by, $w=\left(w_{1}, w_{2}, w_{3}, w_{4}, w_{5}\right)^{T}$

Where

$$
\begin{aligned}
& w_{4}>0 \text { free } \\
& w_{1}=\frac{-\psi x_{1} \theta_{1}\left[(1-f) \alpha+f \theta_{2}+a+\pi\right]}{\theta_{1}\left\{\theta_{1}\left(f \theta_{2}+a+\pi\right)+(a+\pi)\left[(1-f) \alpha+f \theta_{2}+a+\pi\right]\right\}} w_{4} \\
& w_{2}=\frac{-\psi \theta_{1} x_{1}}{\theta_{1}\left(f \theta_{2}+a+\pi\right)+(a+\pi)\left[(1-f) \alpha+f \theta_{2}+a+\pi\right]} w_{4} \\
& w_{3}=\frac{(\eta+a+\pi)}{\delta} w_{4} \\
& w_{5}=\frac{1}{a+\pi}\left\{\eta-\frac{f \theta_{2} \psi x_{1} \theta_{1}}{\theta_{1}\left(f \theta_{2}+a+\pi\right)+(a+\pi)\left((1-f) \alpha+f \theta_{2}+a+\pi\right)}\right\} w_{4} \\
& \text { Similarly, the left eigenvalues is given by } \\
& v=\left(v_{1}, v_{2}, v_{3}, v_{4}, v_{5}\right)^{T} \\
& \mathrm{v}_{1}>0 \text { free } \\
& v_{2}=\frac{\left(\theta_{1}+a+\pi\right) v_{1}}{\theta_{1}}
\end{aligned}
$$

$$
\begin{gathered}
v_{3}=\frac{\psi \delta x_{1} v_{1}}{\psi \delta x_{1}-(\eta+a+\pi)(\delta+a+\pi)} \\
v_{5}=0
\end{gathered}
$$

After computing the right and left eigenvalues, we have theorem (2.5) in [23] to establish the conditions for the existence of backward bifurcation by determining the sign of $a$ and $b$ as indicated in the theorem.

\subsection{Computation of $a$ and $b$}

From the normalised model system (20) the associated non-zero partial derivatives of $F$ at disease free equilibrium are given by

$$
\begin{gathered}
\frac{\partial^{2} f_{1}}{\partial x_{1} \partial x_{4}}=-\psi \\
\frac{\partial^{2} f_{3}}{\partial x_{1} \partial x_{4}}=\psi \\
a=\sum_{k, i, j=1}^{n} v_{k} w_{i} w_{j} \frac{\partial^{2} f_{k}(0,0)}{\partial x_{i} x_{j}} \\
=v_{1} w_{1} w_{4} \frac{\partial^{2} f_{1}}{\partial x_{1} \partial x_{4}}+v_{3} w_{1} w_{4} \frac{\partial^{2} f_{3}}{\partial x_{1} \partial x_{4}} \\
=-v_{1} w_{1} w_{4} \psi+v_{3} w_{1} w_{4} \psi \\
=\frac{\left[(1-f) \alpha+f \theta_{2}+a+\pi\right] x_{1} \theta_{1} w_{4}^{2} \psi^{2}}{\theta_{1}\left\{\theta_{1}\left(f \theta_{2}+a+\pi\right)+(a+\pi)\left[(1-f) \alpha+f \theta_{2}+a+\pi\right]\right\}}\left(v_{1}-v_{3}\right)
\end{gathered}
$$

Thus $a_{0}>0$ if $v_{1}>v_{3}$

$$
\begin{gathered}
b=\sum_{k, i, j=1}^{n} v_{k} w_{i} \frac{\partial^{2} f_{k}(0,0)}{\partial x_{i} \partial \psi} \\
\frac{\partial^{2} f_{1}}{\partial x_{1} \partial \psi}=-x_{4} \\
\frac{\partial^{2} f_{3}}{\partial x_{1} \partial \psi}=x_{4} \\
b=v_{1} w_{1} \frac{\partial^{2} f_{1}}{\partial x_{1} \partial \psi}+v_{3} w_{1} \frac{\partial^{2} f_{3}}{\partial x_{1} \partial \psi} \\
=\frac{\left[(1-f) \alpha+f \theta_{2}+a+\pi\right] \psi x_{1} \theta_{1} x_{4} w_{4}}{\theta_{1}\left\{\theta_{1}\left(f \theta_{2}+a+\pi\right)+(a+\pi)\left[(1-f) \alpha+f \theta_{2}+a+\pi\right]\right\}}\left(v_{1}-v_{3}\right)
\end{gathered}
$$

Thus $b<0$ if $v_{1}<v_{3}$

Then the following theorem must be satisfied. Theorem 3

If $a>0, \mathrm{~b}<0$. when $\psi<0$ with $|\psi| \ll 1,0$ is unstable, there exists a locally asymptotically stable negative equilibrium, when $0<\psi \ll 1,0$ is stable and a positive unstable equilibrium appears.

Figure 2 illustrates a forward bifurcation of the force of infection $\lambda *$ at equilibrium against the basic reproduction number $R_{0}$ of the basic model (6-10). It is observed that as $R_{0}$ increases to one, the disease also increases as a result of 
lack of vaccination occurs when $R_{0}<1$. When $R_{0}=1$, the disease free equilibrium (DFE) and the endemic equilibrium point (EEP) co-exists this implies that the disease cannot be wiped out of the community due to a high endemic level. It is also observed that when $R_{0}>1$ the disease continues to persist. Hence theorem 2 holds.

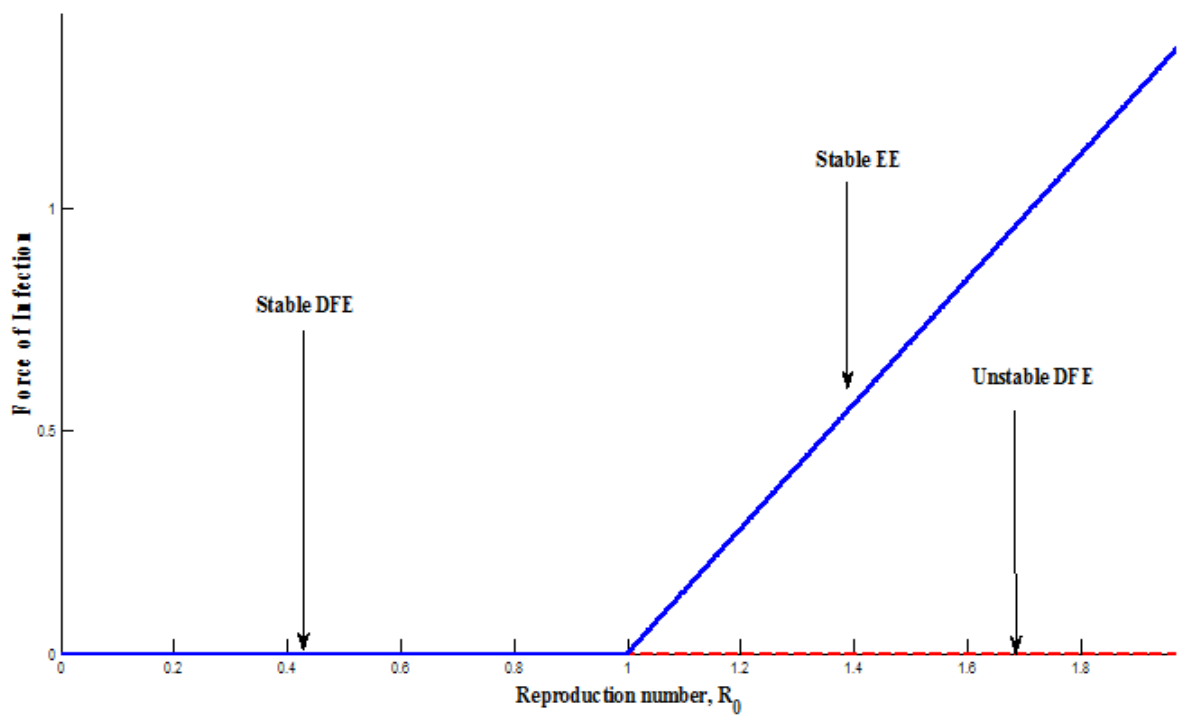

Figure 2. Forward bifurcation at $R_{0}<1$

\subsection{Global Stability of Endemic Equilibrium Point}

Global stability of the EE is explored via the construction of a suitable Lyapunov function. Since the DFE is locally stable this will suggest local stability of the EE for the reverse condition as in [7], we only investigate the global stability of the endemic equilibrium.

Theorem 4

If $R_{0}>1$, then the system has a unique EE point $P *$ which is GAS in $D$.

Proof.

Consider the following function.

$$
\begin{aligned}
V= & A_{1}\left(s-s^{*} \ln s\right)+A_{2}\left(v-v^{*} \ln v\right)+A_{3}\left(e-e^{*} \ln e\right) \\
& +A_{4}\left(i-i^{*} \ln i\right)+A_{5}\left(r-r^{*} \ln r\right) \\
V^{\prime}= & A_{1}\left(1-\frac{s^{*}}{s}\right) s^{\prime}+A_{2}\left(1-\frac{v^{*}}{v}\right) v^{\prime}+A_{3}\left(1-\frac{e^{*}}{e}\right) e^{\prime} \\
& +A_{4}\left(1-\frac{i^{*}}{i}\right) i^{\prime}+A_{5}\left(1-\frac{r^{*}}{r}\right) r^{\prime}
\end{aligned}
$$

If we substitute the expressions for $s^{\prime}, v^{\prime}, e^{\prime}, i^{\prime}, h^{\prime}, r^{\prime}$ from equation (6-10)

We get

$$
\begin{aligned}
V^{\prime}= & A_{1}\left(1-\frac{s^{*}}{s}\right)\left[(1-\Phi) \pi+(1-\rho) a+(1-f) \alpha v-\left(\beta c s i+\theta_{1}+a+\pi\right) s\right] \\
& +A_{2}\left(1-\frac{v^{*}}{v}\right)\left[\Phi \pi+\rho a+\theta_{1} s-\left((1-f) \alpha+f \theta_{2}+a+\pi\right) v\right] \\
& +A_{3}\left(1-\frac{e^{*}}{e}\right)[\beta c s i-(\delta+a+\pi) e]+A_{4}\left(1-\frac{i^{*}}{i}\right)[\delta e-(\eta+a+\pi) i] \\
& +A_{5}\left(1-\frac{r^{*}}{r}\right)\left[\eta i+f \theta_{2} V-(a+\pi) r\right]
\end{aligned}
$$

$$
\begin{aligned}
V^{\prime}= & A_{1}\left(1-\frac{s^{*}}{s}\right)\left[s^{*}\left(\beta c i^{*}+\theta_{1}+a+\pi\right)-\alpha(1-f) v^{*}+\alpha(1-f) v\right. \\
& \left.-s\left(\beta c i+\theta_{1}+a+\pi\right)\right]+A_{2}\left(1-\frac{v^{*}}{v}\right)\left[\left((1-f) \alpha+f \theta_{2}+a+\pi\right) v^{*}\right. \\
& -\left((1-f) \alpha+f \theta_{2}+a+\pi\right) v+\theta_{1} s\left(1-\frac{s^{*}}{s}\right] \\
& +A_{3}\left(1-\frac{e^{*}}{e}\right)\left[\beta c s i-\frac{\beta c s^{*} i^{*}}{e^{*}} e\right]+A_{4}\left(1-\frac{i^{*}}{i}\right)\left[\delta e-\frac{\delta e^{*}}{i^{*}} i\right] \\
& +A_{5}\left(1-\frac{r^{*}}{r}\right)\left[\eta i+f \theta_{2} v-\frac{\left(\eta i^{*}+f \theta_{2} v^{*}\right)}{r^{*}} r\right] \\
V^{\prime} & =A_{1}\left(1-\frac{s^{*}}{s}\right)\left[\beta c\left(s^{*} i^{*}-s i\right)+\left(\theta_{1}+a+\pi\right)\left(s^{*}-s\right)\right. \\
& \left.+\alpha(1-f)\left(v-v^{*}\right)\right]+A_{2}\left(1-\frac{v^{*}}{v}\right)\left[\left((1-f) \alpha+f \theta_{2}+a+\pi\right)\left(v^{*}-v\right)\right. \\
& \left.+\theta_{1}\left(s-s^{*}\right)\right]+A_{3}\left(1-\frac{e^{*}}{e}\right)\left[\beta c s i-\frac{\beta c s^{*} i^{*}}{e^{*}} e\right] \\
& +A_{4}\left(1-\frac{i^{*}}{i}\right)\left[\delta e-\frac{\delta e^{*}}{i^{*}} i\right]+A_{5}\left(1-\frac{r^{*}}{r}\right)\left[\eta i+f \theta_{2} v-\frac{\left(\eta i^{*}+f \theta_{2} v^{*}\right)}{r^{*}} r\right]
\end{aligned}
$$

Further simplification yields

$$
\begin{aligned}
\mathrm{v}^{\prime}= & -A_{1}\left(1-\frac{s^{*}}{s}\right)^{2} s\left(\theta_{1}+a+\pi\right)-A_{2}\left(1-\frac{v^{*}}{v}\right)^{2} v\left[(1-f) \alpha+f \theta_{2}+a+\pi\right] \\
& +A_{1}\left(1-\frac{s^{*}}{s}\right)\left(1-\frac{s}{s^{*} i^{*}} i\right) \beta c s^{*} i^{*}+A_{1}\left(1-\frac{s^{*}}{s}\right)\left(1-\frac{v^{*}}{v}\right) v(1-f) \alpha \\
& +A_{2}\left(1-\frac{v^{*}}{v}\right)\left(1-\frac{s^{*}}{s}\right) \theta_{1} s+A_{3}\left(1-\frac{e^{*}}{e}\right)\left(1-\frac{s^{*} i^{*} e}{s i e^{*}}\right) \beta c s i \\
& +A_{4}\left(1-\frac{i^{*}}{i}\right)\left(1-\frac{e^{*} i}{e i^{*}}\right) \delta e+A_{5}\left(1-\frac{r^{*}}{r}\right)\left(1-\frac{i^{*} r}{i r^{*}}\right) \eta i \\
& +A_{5}\left(1-\frac{r^{*}}{r}\right)\left(1-\frac{v^{*} r}{v r^{*}}\right) f \theta_{2} v
\end{aligned}
$$

Which can in same way be written a

$$
\begin{aligned}
v^{\prime}= & -A_{1}\left(1-\frac{s^{*}}{s}\right)^{2} s\left(\theta_{1}+a+\pi\right)-A_{2}\left(1-\frac{v^{*}}{v}\right)^{2} v[(1-f) \alpha \\
& \left.+f \theta_{2}+a+\pi\right]+F(s, v, e, i, r)
\end{aligned}
$$


where

$$
\begin{aligned}
F(s, v, e, i, r)= & A_{1}\left(1-\frac{s^{*}}{s}\right)\left(1-\frac{s}{s^{*} i^{*}} i\right) \beta c s^{*} i^{*}+A_{1}\left(1-\frac{s^{*}}{s}\right)\left(1-\frac{v^{*}}{v}\right) v(1-f) \alpha \\
& +A_{2}\left(1-\frac{v^{*}}{v}\right)\left(1-\frac{s^{*}}{s}\right) \theta_{1} s+A_{3}\left(1-\frac{e^{*}}{e}\right)\left(1-\frac{s^{*} i^{*} e}{s i e^{*}}\right) \beta c s i \\
& +A_{4}\left(1-\frac{i^{*}}{i}\right)\left(1-\frac{e^{*} i}{e i^{*}}\right) \delta e+A_{5}\left(1-\frac{r^{*}}{r}\right)\left(1-\frac{i^{*} r}{i r^{*}}\right) \eta i \\
& +A_{5}\left(1-\frac{r^{*}}{r}\right)\left(1-\frac{v^{*} r}{v r^{*}}\right) f \theta_{2} v
\end{aligned}
$$

where $F$ is non-positive using a modified version of [24],[25] and[26].Thus, $F \leq 0$ for some $s, v, e, i, r>0$. Hence $\frac{d V}{d t} \leq 0$ for all $s, v, e, i, r$ and is zero when $s=s^{*}, v=v^{*}, e=e^{*}, i=i^{*}, r=r^{*}$.

Therefore, the largest compact invariant set in $D$ such that $\frac{d V}{d t}=0$ is the singleton $\{p *\}$ which is the endemic equilibrium point. Invariant principle of [27] guarantee that $p *$ is globally asymptotically stable (GAS) is $\stackrel{0}{D}$, the interior of $D$. Thus we have proved theorem 4 .

\section{Simulation and Discussion}

The main objective of this study was to assess the impact of immunization strategies on the transmission dynamics of the disease. In order to support the analytical results, graphical representations showing the variations in parameters with respect to different state variables have been presented in this section.

Since, most of the parameters were not readily available; it was found convenient to estimate them just for illustrations purposes on how the model would behave in different real situations. Table 2 shows the set of parameter values which were used in the simulation.

Table 2. Parameters used in model simulations

\begin{tabular}{lll}
\hline Parameter & Value & Source \\
\hline$f$ & 0.5 & Estimated \\
$\pi$ & $0.45 /$ year & Estimated \\
$\theta_{1}$ & $0.7 /$ year & Estimated \\
$\theta_{2}$ & $0.8 /$ year & Estimated \\
$\alpha$ & $0.36 /$ year & Estimated \\
$\rho$ & 0.7 & Estimated \\
$\eta$ & $0.6 /$ year & Estimated \\
$\delta$ & $0.3 /$ year & Estimated \\
$a$ & $0.2 /$ year & Estimated \\
\hline
\end{tabular}

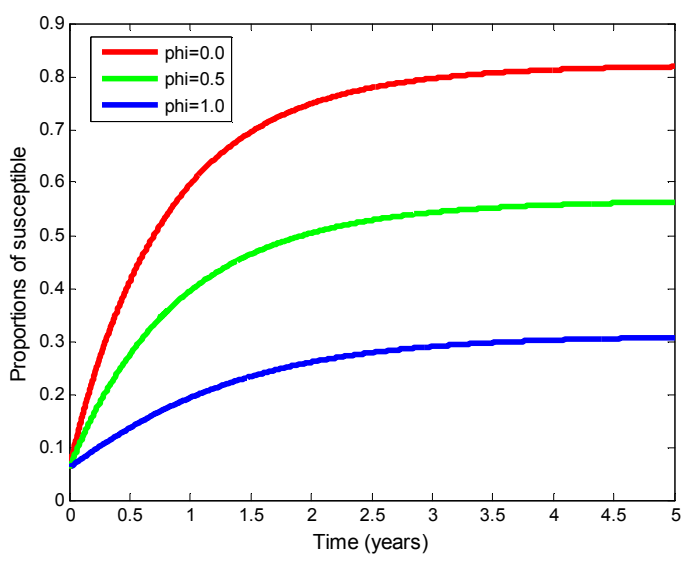

Figure 3. Susceptible population in an outbreak, varying the proportion of newborns vaccinated $(p h i=0.0,0.5,1)$

Figure 3 shows simulations with different proportions of the newborns vaccinated. As the outbreak occurs later, the vaccination campaign starts to produce effects, decreasing the total number of susceptible humans; such a decrease in susceptible humans will automatically render to a decrease of the sick humans and hence control the disease eruption.

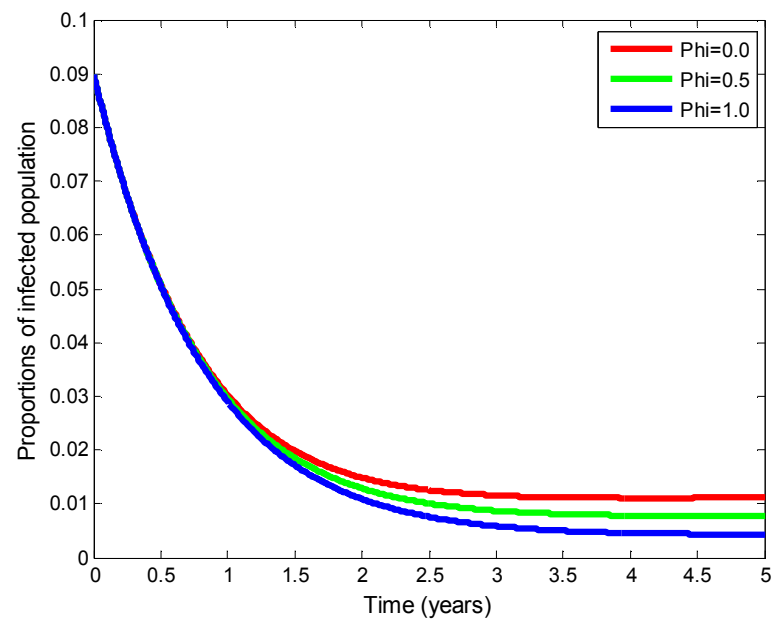

Figure 4. Infected population in an outbreak, varying the proportion of newborns vaccinated $(p h i=0.0,0.5,1)$

Figure 4 shows simulations with different proportions of the newborns vaccinated. As the outbreak occurs later, the vaccination campaign starts to produce effects, decreasing the total number of infected humans; such a decrease in sick humans will automatically render to eradication of the VZV from the community. Vaccination campaign centered in newborns is a best for the future of a country, but does not produce instantly results to fight the disease. To achieve immediate results, it is necessary to use random mass vaccination, which means that it is necessary to vaccine a significant part of the population. 


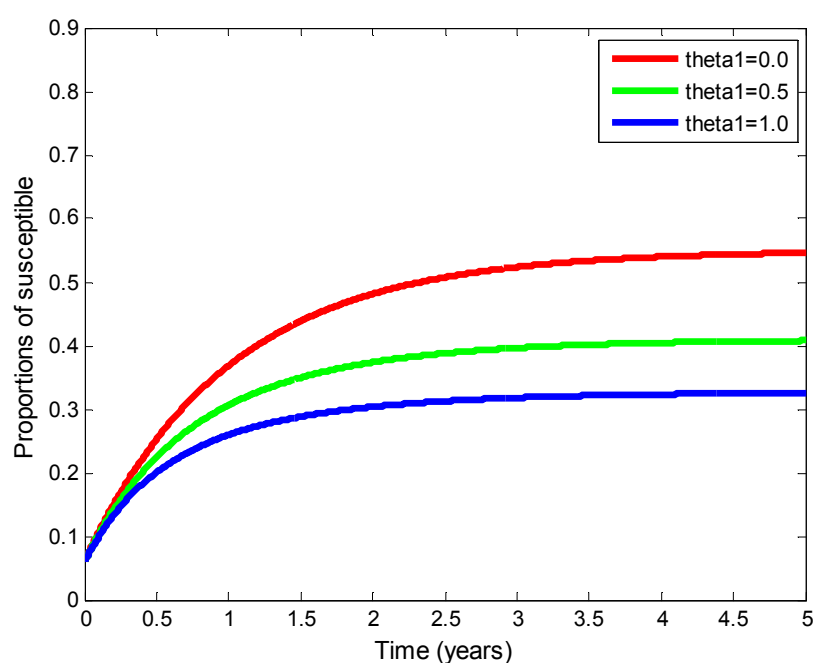

Figure 5. Susceptible population in an outbreak, varying the proportion of susceptible vaccinated (theta $1=0.0,0.5,1$ )

It can be seen from Figure 5 that, more increase in vaccination proportion of susceptible adults, tend to reduce the proportion of susceptible and as a result reduction in number of sick humans and hence chickenpox diminishes.

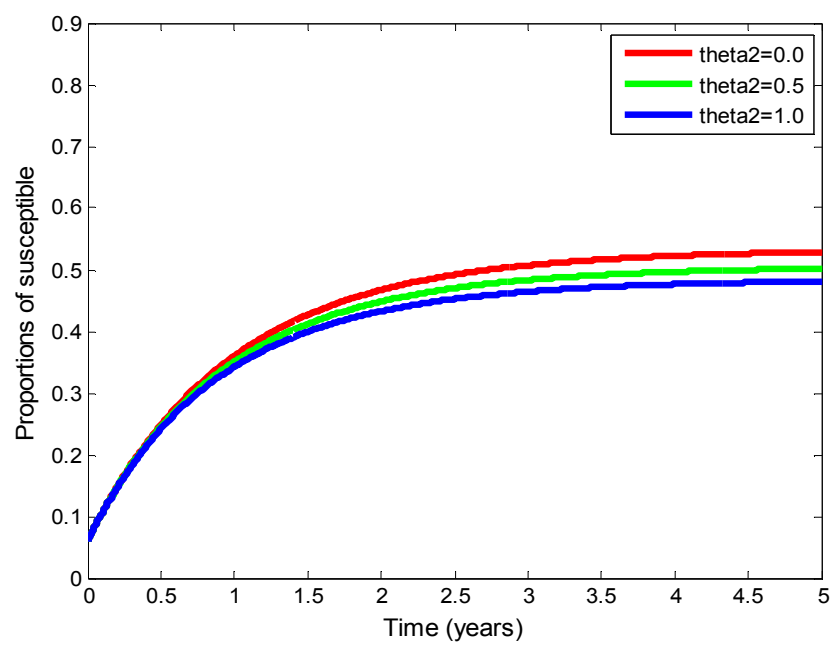

Figure 6. Susceptible population in an outbreak, varying the dose two coverage (theta $2=0.0,0.5,1)$.

It can be seen from Figure 6 that the more increase in the coverage of dose two among the humans ,is the more the reduction in the proportion of susceptible but in a small amount this is due to the fact that susceptible humans are not directly related to individual who receive second dose , susceptible humans will receive dose two only if they had already received dose one otherwise they do not receive dose two such a condition is what made a slight decrease in susceptible population even when more individuals are vaccinated in dose two because such people might be newborns or recruits hence not or less affecting population of susceptible. So in general this practice has less but significant impact in reducing the disease.

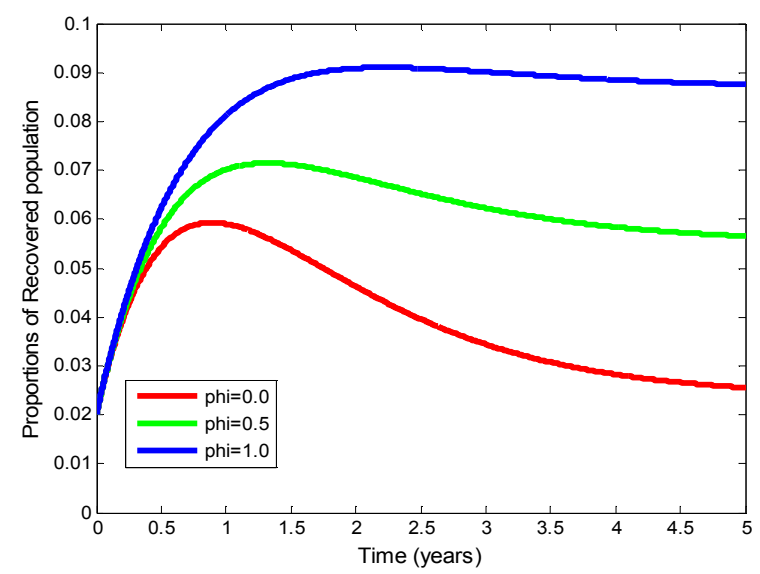

Figure 7. Recovered population in an outbreak, varying the proportion of newborns vaccinated $(p h i=0.0,0.5,1)$

It can be seen from Figure 7 that the number of recovered individuals increases with increase in the vaccination coverage of newborns, it can be seen that when no newborns are vaccinated there is increase in the recovered populations, this might be due to natural immunity of the sick ones, and a corresponding decline is perhaps due to loss of immunity which in fact wanes with time. In the Figure 7, with the graph colored green, it can be noted that with a $50 \%$ of newborns being vaccinated, there is a significant increase in the population of recovered humans. However as time increases we note a slight decline in the recovered humans, this agrees with our intuition that the first vaccine wanes with time this calls for the next boosting up vaccine coverage. The top most graph, colored blue of Figure 7 shows the maximum proportion of recovered humans, when the vaccination coverage is $100 \%$,we see that the graph is increasing and retain almost constant after reaching the maximum point this suggest that when vaccination coverage is optimal then the disease can be eradicated from the community.

\section{Sensitivity Analysis}

Sensitivity analysis is used to determine how "sensitive" a model is to changes in the value of the parameters of the model and to changes in the structure of the model. Sensitivity analysis helps to build confidence in the model by studying the uncertainties that are often associated with parameters in models. Sensitivity indices allow us to measure the relative change in a state variable when a parameter changes. Sensitivity analysis is commonly used to determine the robustness of model predictions to parameter values (since there are usually errors in data collection and presumed parameter values). Thus we use it to discover parameters that have a high impact on $\mathrm{R}_{0}$ and should be targeted by intervention strategies. If the result is negative, then the relationship between the parameters and $\mathrm{R} 0$ is inversely proportional. In this case, we will take the modulus of the sensitivity index so that we can deduce the size of the effect of changing that parameter. On the other hand, a positive sensitivity index means an increase in the 
value of a parameter.

The explicit expression of $R_{0}$ is given by the equation 14 . Since $\mathrm{R}_{0}$ depends only on six parameters, we derive an analytical expression for its sensitivity to each parameter using the normalized forward sensitivity index as by [28] as follows:

$$
\begin{gathered}
\Upsilon_{c}^{R_{0}}=\frac{\partial R_{0}}{\partial c} \times \frac{c}{R_{0}}=+1 \\
\Upsilon_{\beta}^{R_{0}}=\frac{\partial R_{0}}{\partial \beta} \times \frac{\beta}{R_{0}}=+1 \\
\Upsilon_{\delta}^{R_{0}}=\frac{\partial R_{0}}{\partial \delta} \times \frac{\delta}{R_{0}}=+0.6842
\end{gathered}
$$

The rest of sensitivity indices for all parameters used in equation (14) can be computed in the similar approach. Table 3 shows the sensitivity indices of $R_{0}$ with respect to the six parameters.

Table 3. Sensitivity indices of $R_{0}$ with respect to each parameter

\begin{tabular}{ll}
\hline Parameter & Sensitivity Index \\
\hline$c$ & +1 \\
$\beta$ & +1 \\
$\delta$ & 0.6842 \\
$\eta$ & -0.4800 \\
$a$ & -0.3344 \\
$\pi$ & -0.7524 \\
\hline
\end{tabular}

From Table 3, we can obtain ' $\Upsilon_{c}^{R_{0}}={ }^{\prime} \Upsilon_{\beta}^{R_{0}}=+1$, this means that an increase in $c$ or $\beta$ will cause an increase of exactly the same proportion in R0. Similarly, a decrease in $\mathrm{c}$ or $\beta$ will cause a decrease in R0, as they are directly proportional. We can also note that $\eta$ or $a$ or $\pi<0$ hence these parameters are inversely proportional to $\mathrm{R} 0$.

It can be seen that, the most sensitive parameters are $c$ and $\beta$ followed by per followed by $\pi$ then $\delta$, then $\eta$ and the least sensitive parameter is $a$.

Therefore, to minimize VZV transmission in a population, this study recommends that, the combination of vaccination and treatment should be implemented. This is due to the fact that, vaccination reduces the likelihood of an individual to be infected, treatment of latently infected people reduces the progression rate to infectious stage and treatment of infectious people will stop them from transmitting the disease.

\section{Conclusion}

In this paper, we have formulated a deterministic mathematical model for transmission dynamics of VZV with vaccination. From the model we have derived the effective reproduction number from which we have deduced the basic reproduction number. The effective reproduction number computed has been used to measure the relative impact for individual or combined intervention for effective disease control. We have derived both the Disease Free Equilibrium (DFE) and the Endemic Equilibrium points (EE) and proved that the DFE is locally asymptotically stable (l.a.s) when $R_{e}<1$ and the EE point is Globally Asymptotically Stable (G.A.S). In the absence of disease-induced death, we prove that the transcritical bifurcation at $R_{0}=1$ is supercritical (forward).

We have performed sensitivity analysis on the basic reproduction number from which we have noted that the most sensitive parameters are the probability of transmission of the disease from an infectious individual to a susceptible individual per contact, $\beta$, per capita contact rate , $c$, per capita birth rate, $\pi$ and the progression rate from latent to infectious stage, $\delta$. Numerical simulations of the model have shown that, the combination of vaccination and treatment is the most effective way to combat the epidemiology of VZV in the community.

\section{Acknowledgments}

I would like to express my sincere gratitude with to The Nelson Mandela Institution of Science and Technology for financial assistance and the University of Dodoma for granting me a study leave.

\section{References}

[1] Almuneef, M., Memish, Z.A., Balkhy, H. H., Alotaibi, B., \& Helmy, M. (2006). Chickenpox Complications in Saudi Arabia: Is it time for routine Vaccination? International $\begin{array}{llll}\text { Journal of InfectiousDiseases.10 (2):156-161. } & \text {. }\end{array}$ http://dx.doi.org/10.1016/j.ijid.2005.02.008

[2] Gershon, A. A. (2008). Varicella-zoster Virus Infections. Pediatr Rev. 29(1):5-10. http://dx.doi.org/10.1542/pir.29-1-5

[3] Vyse, A. J., Gay, N. J., Hesketh, L. M., Morgan- Capner, P., \& Miller, E.(2004). Seroprevalence prevalence of antibody to varicella zoster virus in England and Wales in children and young adults. Epidemiol Infect. 132(6):1129-1134. http://dx.doi.org/10.1017/S0950268804003140

[4] Fairley, C. K., \& Miller, E. (1996). Varicella-zoster virus epidemiology--a changing scene? J Infect Dis.174 (Suppl 3):314-319.http://dx.doi.org/10.1093/infdis/174.

Supplement_3.S314

[5] Lee, B.W. (1998).Review of Varicella Zoster Seroepidemiology in India and Southeast Asia. Trop Med Int Health.3(11): 886-90. http://dx.doi.org/10.1046/j.13653156.1998.00316.x

[6] Diekman,O., Heesterbeek, J.A.P. and Metz, J.A.P. (1990).On the definition and Computation of the basic reproduction ratio $R_{0}$ in the model of infectious disease in Heterogeneous populations. Journal of Mathematical Biology. 2(1):265-382.

[7] Garnett ,G.P and Greenfell,B. T.(1992). The epidemiology of varicella-zoster virus infections: a mathematical model. Epidemiol. Infect.108, 495-511 
[8] Furguson, N.M, Anderson ,R.M and Garnett, G.P(1996).Mass vaccination to control chickenpox: The influence of zoster(population dynamics/infectious diseases/mathematical

modeling/varicella/immunization).Medicinal sciences.93, $7231-7235$

[9] Mathew ,C.S and Herbert, W.H(1999).Modeling the Effects of Varicella Vaccination Programs on the Incidence of Chickenpox and Shingles. Bulletin of Mathematical Biology . 61, 1031-1064

[10] Gershon, A. A., P. LaRussa, S. Steinberg, N. Mervish, S. H. Lo and P. Meier (1996). The protective effect of immunologic boosting against zoster: an analysis in leukemic children who were vaccinated against chickenpox. J. Infect. Dis. 173, 450-453.

[11] Hope-Simpson, R. E. (1965). The nature of herpes zoster: a long term study and a new hypothesis. Proc. Roy. Soc. Med. $58,9-20$.

[12] Centers for Disease Control and Prevention (1997). Prevention of varicella: recommendations of the Advisory Committee on Immunization Practices (ACIP). MMWR 45(No. RR-11), 35-40.

[13] Chaves SS, Zhang J, Civen R, Watson BM, Carbajal T, et al. (2008) Varicella disease among vaccinated persons: clinical and epidemiological characteristics,1997-2005. J Infect Dis 197 Suppl 2: S127-131.

[14] Levin MJ, Smith JG, Kaufhold RM, Barber D, Hayward AR, et al. (2003).Decline in varicella-zoster virus (VZV)-specific cell-mediated immunity with increasing age and boosting with a high-dose VZV vaccine. J Infect Dis 188:1336-1344.

[15] Berger R, Florent G, Just M (1981) Decrease of the lymphoproliferative response to varicella-zoster virus antigen in the aged. Infect Immun 32: 24-27.

[16] Miller AE (1980) Selective decline in cellular immune response to varicella-zoster in the elderly. Neurology 30 : $582-587$.

[17] Poletti P, Melegaro A, Ajelli M, del Fava E, Guzzetta G, et al. (2013) Perspectives on the Impact of Varicella Immunization on Herpes Zoster. A Model-Based Evaluation from Three European Countries. PLoS ONE 8(4): e60732. doi:10.1371/journal.pone.0060732
[18] Van den Driessche,P and Watmough, J(2002). Reproduction numbers and sub-threshold endemic equilibria for compartmental models of disease transmission. Mathematical Biosciences. 180(1-2):29-48.

[19] Castillo-Chavez,C Feng.Z and W. Huang (2002).On the Computation of R0 and Its Role on Global Stability, In: Mathematical Approaches for Emerging and Reemerging Infectious Diseases: An Introduction, Springer-Verlag, NewYork: 229- 250. doi:10.1007/978-1-4613-0065-6

[20] Nakul C, Cushing JM, Hyman JM (2006). Bifurcation Analysis of Mathematical model for malaria transmission. SIAM J. Appl. Math. 67(1): 24 - 45.

[21] Dushoff J, Huang W, Castillo-Chavez C (1998). Backwards bifurcations and catastrophe in simple models of fatal diseases.J. Math. Biol.36:227-248. [PubMed: 9528119]

[22] Gumel, A.B., Song, B., 2008. Existence of multiple-stable equilibria for a multi-drug-resistant model of mycobacterium tuberculosis. Math. Biosci. Eng. 5 (3), $437-$ 455.

[23] Castillo-Chavez, C., Song, B (2004).Dynamical Models of Tuberculosis and their Applications. Mathematical Biosciences and Engineering 1(2):361-404.and ReEmerging Infectious Diseases: An Introduction, Springer Verlag.

[24] Barbalat I (1959).Systeme d'e quationdiff e rentielle d'Oscillation, Nonlin e aires. Rev Roum Math Pures Appl $4: 267-270$

[25] Mukandavire Z, Garira W (2006) .HIV/AIDS model for assessing the effects of prophylactic sterilizing vaccines, condoms and treatment with amelioration. $J$ Biol Syst .14(3):323-355

[26] McCluskey, C.C.(2006) Lyapunov functions for tuberculosis models with fast and slow progression. Math Biosci Eng $3(4): 603-614$

[27] LaSalle JP (1976) .The stability of dynamical systems, CBMS-NSF regional conference series in applied mathematics 25. SIAM, Philadelphia.

[28] Chitnis, N., Hyman, J.M., and Cusching, J. M. (2008). Determining important Parameters in the spread of malaria through the sensitivity analysis of a mathematical Model. Bulletin of Mathematical Biology 70(5):1272-12 